\section{Beating diffraction in the far field}

Diffraction makes it impossible to resolve those features of an object that are smaller than the wavelength of the light used to probe it. Yet this most basic principle of optics - known as - has its exceptions. Perhaps the most well-known is that it applies only to images produced in the 'far field', some distance away from the object. Consequently, it is possible to resolve the diffraction limit subwavelength features in an object by scanning a probe (such as the end of an optical fibre) through the optical field in the immediate vicinity of an object bathed in light - which is how a near-field scanning optical microscope works.

Two groups may exception (Opt.Express 14, 8247-8256; 2006 and Phys. Rev. B 74, 075103; 2006). They propose have found another that a cylindrical 'hyperlens', whose radial and tangential permittivities are opposite in sign, should convert evanescent waves that carry information about an object's fine structure into waves propagating into the far field. Such far-field waves could then be manipulated with conventional optics to reveal the subwavelength features within the structure of an object.

\section{THE SIMPLEST QUANTUM GAS}

Hydrogen is the most abundant element in the Universe but it's relatively scarce on Earth, where it exists as a diatomic gas, $\mathrm{H}_{2}$, that easily escapes the atmosphere. Atomic hydrogen is even rarer, as atoms tend to recombine into $\mathrm{H}_{2}$. In 1997, a two-dimensional gas of hydrogen atoms adsorbed on a film of superfluid 'He was seen to form a 'local' Bose-Einstein condensate. Now Janne Ahokas show that a solid $\mathrm{H}_{2}$ film can also trap hydrogen atoms and that the resulting gas is stable for up to two weeks (Phys. Rev. Lett.97, 095301; 2006).

At $150 \mathrm{mK}$, the gas of these trapped hydrogen atoms has a density of order $10^{18} \mathrm{~cm}^{-3}$. Electron-spin-resonance spectra show that the hydrogen atoms have a reduced hyperfine interaction relative to free atoms, suggesting that the atoms are located at substitutional sites in the place of host molecules - within the $\mathrm{H}_{2}$ crystals.

Intriguingly, the populations of the two lowest hyperfine states do not follow Boltzmann statistics; rather, a large population occupies the ground state. Further studies will confirm whether it is a Bose-Einstein condensate.

\section{Bacterial swim team}

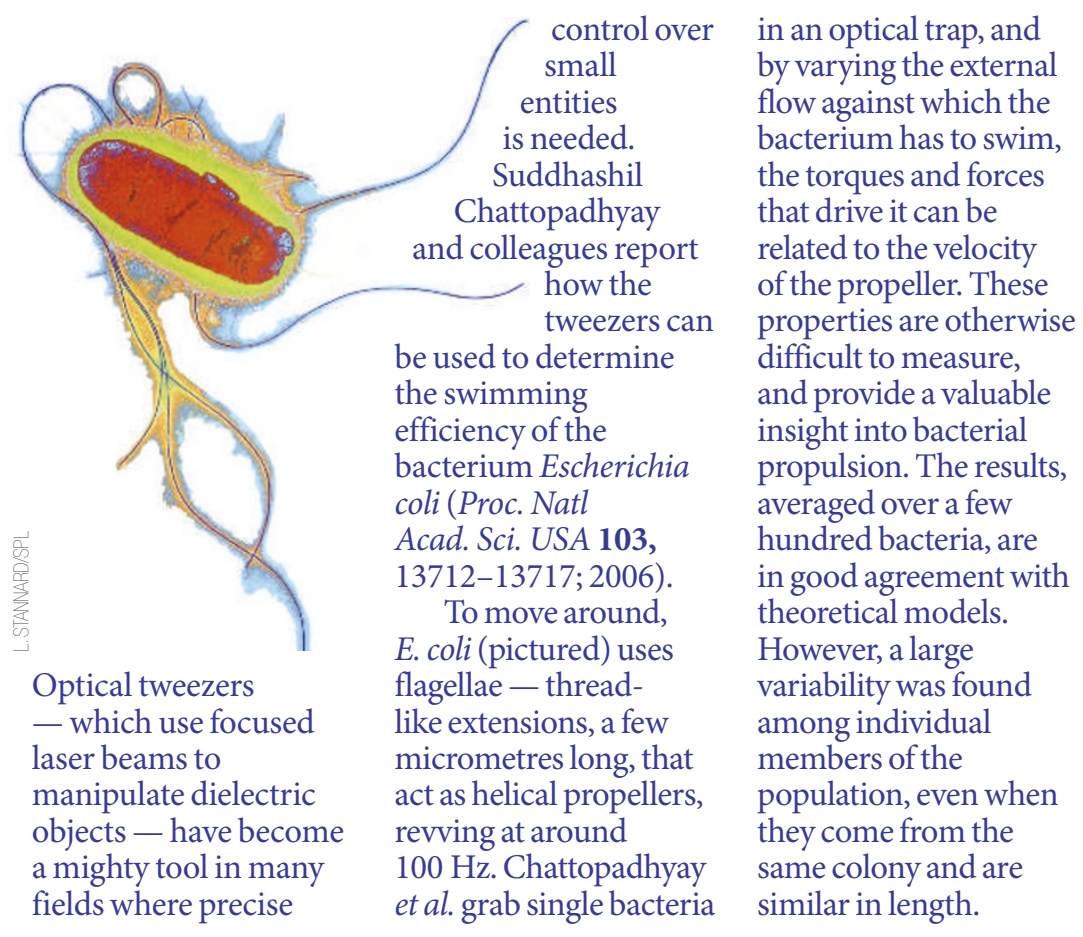

\title{
Light condensates
}

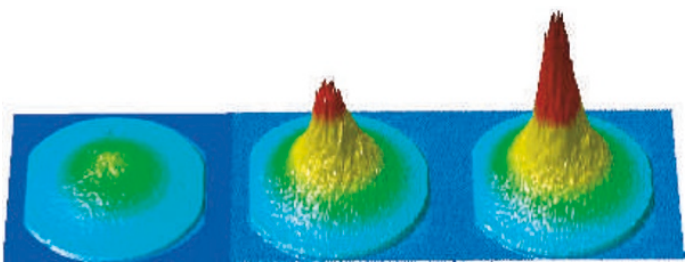

When bosons are so slow that their de Broglie wavelength becomes comparable to the distance between particles, a Bose-Einstein condensate (BEC) forms. This state of matter is characterized by a massive occupation of the lowest quantum state, leading to coherence between the particles on a macroscopic scale, which, famously, has been observed in dilute atomic gases, at temperatures below a microkelvin. Now, Jacek Kasprzak and colleagues present the hallmarks of a BEC in a solid-state system, and at temperatures of several Kelvin (Nature 443, 409-414; 2006).
This condensate is formed from so-called exciton polaritons. These are bosonic quasiparticles, part light, part matter, created by the strong coupling of electronic excitations in a semiconductor (excitons) to photons in an optical microcavity. The mass of an exciton polariton is 10,000 times smaller than that of a free electron and this drastically relaxes the temperature and density criteria for achieving Bose-Einstein condensation. Hence, in this system, prominent lowest-state occupation (pictured) could be seen at above-liquid-helium temperatures, on increasing the particle density.

\section{Reaction dynamics}

The drive to monitor chemical reactions in real-time has led to femtosecond spectroscopy, using thousands of successive laser pulses. But reaction products, as well as degradation of the sample, often get in the way. To counter this, Peter Poulin and Keith Nelson take one laser pulse and apply a spatial gradient - two orthogonal glass echelon structures with 20 steps each - to introduce a time delay such that multiple pulses hit the sample at different times (Science doi:10.1126/ science.1127826; 2006). Thus, from a single pump-laser pulse, 400 probe pulses reach the sample with 25 -fs relative delays.

Their technique is particularly useful for irreversible or catastrophic reactions. As a demonstration, they studied three molecular crystals that contain triiodide $\left(\mathrm{I}_{3}{ }^{-}\right)$in different lattice environments. On light absorption, $\mathrm{I}_{3}{ }^{-}$dissociates to a bound $\mathrm{I}_{2}{ }^{-}$and neutral I. Because each laser pulse samples a fresh region of the crystal, the discolouration does not affect subsequent pulses. And the $\mathrm{I}_{2}^{-}$absorption spectra, unaffected by interference from $\mathrm{I}_{3}$ absorption, showed clear evidence for crystal lattice effects on the reaction dynamics. 